\title{
A Comparative Study of Platelet Parameters in Non-Diabetic Chronic Kidney Disease Stage 3,4 and End Stage Renal Disease on Maintenance Haemodialysis
}

\author{
P. Mohanraj ${ }^{1}$, Dulla Nageswara Rao² \\ ${ }^{1}$ Associate Professor, Department of Medicine, Meenakshi Medical College Hospital Research Institute, Kanchipuram, ${ }^{2}$ Post \\ Graduate Student, Department of Medicine, Meenakshi Medical College Hospital \& Research Institute, Kanchipuram, India
}

Corresponding author: P. Mohanraj, New No 13, Old No 5, Thiruvalluvar Street, Little Kanchipuram, Kanchipuram-631501, India

DOI: http://dx.doi.org/10.21276/ijcmsr.2019.4.3.1

How to cite this article: P. Mohanraj, Dulla Nageswara Rao. A comparative study of platelet parameters in non-diabetic chronic kidney disease stage 3,4 and end stage renal disease on maintenance haemodialysis. International Journal of Contemporary Medicine Surgery and Radiology. 2019;4(3):C1-C5.

\section{A B S T R A C T}

Introduction: Chronic Kidney Disease (CKD) represents a condition with very high cardio vascular risk and by an increase in pro-oxidant and pro-inflammatory mediators in the bloodstream, progressively enhances the athero-thrombotic processes as the renal function decreases. The platelet parameters are studied in patients with coronary artery disease along with risk factors like diabetes, hypertension and CKD. However contrasting results have been reported so far on the effects of declining renal function on platelet volume. Aim: The aim of our study is to investigate the relationship between Platelet parameters and glomerular filtration rate (GFR) in patients with non-diabetic CKD and whether altered Platelet parameters were correlated with worsening of renal function.

Material and methods: This study was performed on 101 subjects into three groups. Control group (Group 1$) n=25, C K D$ stage 3 and 4(Group 2) n=46, CKD stage 5 (Group 3) n=30 on maintenance Haemodialysis include both the gender in the age group of 18 to 65 years. After the exclusion of diabetes, prior history of CVA, CAD, obesity and smoking, patients were informed about the study pattern, and blood sample were drawn for the measurement of platelet parameters.

Results: The mean MPV of group 2 and group 3 patients were $10.62 \pm 1.76$ and $10.06 \pm 2.08$ respectively $(P<0.001)$. Similarly PDW and P-LCR of group 2, 3 patients were $14.67 \pm 1.89,14.56 \pm 1.80(P<0.001)$ and $31.35 \pm 7.16,24.14 \pm 5.44$ $(P<0.001)$ respectively. The mean Platelet count of group 2 and 3 patients were $2.44 \pm 0.84$ and $1.93 \pm 0.78(P>0.001)$ respectively.

Conclusion: In our study the MPV as an indicator of increased platelet activity was significantly increased in CKD stage 3, 4 and 5, when compared to general population, but at the same time it was not significantly altered by Haemodialysis.

Keywords: Chronic Kidney Disease, Platelet parameters, MPV.

\section{INTRODUCTION}

Coronary artery disease (CAD) still represents the leading cause of mortality worldwide. ${ }^{1}$ Chronic kidney disease (CKD) represents a condition with a very high cardiovascular risk, both mediated by the association with main established risk factors, such as diabetes, hypertension, dyslipidaemia and by an increase in pro-oxidant and proinflammatory mediators in the bloodstream, progressively enhancing the atherothrombotic processes as the renal function declines. ${ }^{2-4}$ The mean platelet volume (MPV), Platelet count, Platelet distribution width (PDW) and Platelet large cell ratio (P-LCR) those are inexpensively and routinely measured by automated cell counters, is an attractive index for research in clinical settings because of its widespread availability. ${ }^{5}$ Larger platelets are activated more easily than smaller platelets by adenosine diphosphate, collagen, and epinephrine and release greater quantities of thromboxane A2, platelet factor 4, and thromboglobulin. ${ }^{6}$ Abnormalities in platelet parameters like mean platelet volume (MPV), platelet distribution width (PDW),platelet count and platelet large cell ratio(PLCR) are studied extensively in patients with coronary artery disease (CAD) and cerebrovascular disease(CVD), both in general population as well as patients with risk factors like diabetes mellitus (DM),hypertension and chronic kidney disease (CKD) ${ }^{7-8}$ However, despite the strict association of $\mathrm{CKD}$ and $\mathrm{CAD}$, and the potential increase in platelet size in patients with cardiovascular disease, contrasting results have been reported so far on the effects of renal function on platelet volume., ${ }^{9,10}$ In the present study, we aimed to investigate the relationship between Platelet parameters and the glomerular filtration rate (GFR) in patients with non-diabetic CKD and whether an altered Platelet parameters were correlated with worsening of renal function. 


\section{MATERIAL AND METHODS}

This study was performed on 101 subjects into three groups. Control group (Group 1), CKD stage 3 and 4 (Group 2), $\mathrm{CKD}$ stage 5 (group 3 ), include both the gender, in the age group of 18 to 65 years during the period of February 2018 to October 2018. Case group included 76 patients with $\mathrm{CKD}$ stage 3 to 5 undergoing medical management and maintenance haemodialysis for more than 6 months at Meenakshi Medical College Hospital and Research Institute. Every patient was informed about the study pattern and the volunteers given consent for participation in this study was included. During the study period no drug was additionally given or not modified.

All the patients of stage $5 \mathrm{CKD}$ (ESRD) are undergoing regular haemodialysis for 3 times a week lasting for 4 hours, using bicarbonate buffer with blood flow of $250 \mathrm{ml}$ per/min and dialysate flow of $500 \mathrm{ml} / \mathrm{min}$ with 1.6 square metre surface area hollow fibre polysulphone membrane dialyser. Control group included 25 subjects of healthy adults including male and female volunteers not having any kidney damage.

\section{Exclusion criteria}

Patient's blood sugar values are evaluated according to ADA diagnostic criteria to exclude the diagnosis of diabetes mellitus. The patient with $\mathrm{HbA} 1 \mathrm{c}$ value of $>6 \% \mathrm{FBS}$ of $>100 \%$ PPBS of $>140$ were excluded from the study. Patients with prior history of CVA, CAD, obesity, smoking and previous long-term drug intake of NSAIDS, anti-platelets and anti-coagulants, hepatic impairment were excluded from both case group and control group.

During prehaemodialysis period in ESRD patients and in all others participants of this study $5 \mathrm{ml}$ of venous blood sample was withdrawn in their 12 hours fasting state. The blood samples were processed by fully automated bidirectional analyser by the method of flow cytometry and hydrodynamic focussing techniques for the measurement of platelet parameter like PDW, MPV, P-LCR, platelet count. With creatinine, GFR was measured with MDRD formula.

\section{MDRD Study Equation}

eGFR(estimated glomerular filtration rate) $=175 \mathrm{x}$ (standardized Serum creatinine $)^{-1.154} \mathrm{x}(\text { age })^{-0.203} \times 0.742$ [if female] x1.212 [if Black]

eGFR expressed in $\mathrm{mL} / \mathrm{min} / 1.73 \mathrm{~m}^{2}$,Standardized Serum Creatinine expressed in $\mathrm{mg} / \mathrm{dL}$ and age expressed in years

\section{STATISTICAL ANALYSIS}

The IBM SPSS, version 22 software tool was used for the data processing. All the values were expressed as mean \pm standard deviation unless otherwise indicated. For correlation analysis, Pearson's correlation test was used when data were suitable to normal distribution. The differences in the mean values between the groups were analyzed by using ANOVA test. A $\mathrm{p}$-value of $<0.05$ was considered statistically significant.

\section{RESULTS}

Demographic and biochemical data of study group are provided in Table 1. Mean ages of the patients in CKD stage 3 and 4(Group 2), CKD stage 5(Group 3) and healthy control (Group 1) groups were $52.67 \pm 8.07,50.43 \pm 10.33$ and 48.56 \pm 11.05 years respectively ( $P$ not significant). Percentages of the female subjects in Group 1, Group 2 and Group 3 were $36 \%, 39 \%$ and $37 \%$, respectively ( $P$ not significant).

Our study showed a statistically significant difference between Group 1,Group 2 and Group 3 in Systolic Blood Pressure, Diastolic Blood Pressure, Urea, Creatinine, Glomerular Filtration Rate $(P<0.01, P<0.01, P<0.01, P<0.01, P<0.01$, respectively).Although the Platelet Count values were lower in CKD group than control group, difference was not statistically significant $(P=0.022)$.

The paired variables SBP and urea in group 1 shows statistical significance, similarly SBP and creatinine in group $1, \mathrm{SBP}$ and DBP in group $3, \mathrm{DBP}$ and urea in group $3, \mathrm{DBP}$ and platelet count in group 3, platelet count and $\mathrm{P}-\mathrm{LCR}$ in group 3 shows statistical significance.

\begin{tabular}{|c|c|c|c|c|}
\hline Parameters & Group $1(n=25)$ & Group $2(n=46)$ & Group $3(n=30)$ & $p$-value \\
\hline \multicolumn{5}{|l|}{ Gender } \\
\hline Females [n (\%)] & $9(36 \%)$ & $18(39 \%)$ & $11(37 \%)$ & \multirow[t]{2}{*}{$0.013^{*}$} \\
\hline Males [n (\%)] & $16(64 \%)$ & 28 (61\%) & $19(63 \%)$ & \\
\hline Age & $48.56 \pm 11.05$ & $52.67 \pm 8.07$ & $50.43 \pm 10.33$ & 0.213 \\
\hline SBP & $129.04 \pm 12.53$ & $152.00 \pm 14.68$ & $147.47 \pm 13.94$ & $<0.001^{* *}$ \\
\hline DBP & $84.08 \pm 3.58$ & $97.26 \pm 9.73$ & $90.80 \pm 8.31$ & $<0.001^{* *}$ \\
\hline urea & $28.60 \pm 7.90$ & $55.58 \pm 19.14$ & $47.87 \pm 16.83$ & $<0.001^{* *}$ \\
\hline $\mathrm{Cr}$ & $0.95 \pm 0.17$ & $2.39 \pm 0.70$ & $5.90 \pm 1.22$ & $<0.001^{* *}$ \\
\hline GFR & $81.16 \pm 22.15$ & $28.20 \pm 9.24$ & $10.57 \pm 2.46$ & $<0.001^{* *}$ \\
\hline MPV & $8.72 \pm 1.86$ & $10.62 \pm 1.76$ & $10.06 \pm 2.08$ & $<0.001^{* *}$ \\
\hline PDW & $12.08 \pm 1.90$ & $14.67 \pm 1.89$ & $14.56 \pm 1.80$ & $<0.001^{* *}$ \\
\hline $\mathrm{PI}$ count & $2.45 \pm 0.90$ & $2.44 \pm 0.84$ & $1.93 \pm 0.78$ & $0.022^{*}$ \\
\hline P-LCR & $32.74 \pm 7.54$ & $31.35 \pm 7.16$ & $24.14 \pm 5.44$ & $<0.001 * *$ \\
\hline
\end{tabular}

*: There exists statistically significant difference in means of different groups of the variables at $5 \%$ level. ${ }^{* *}$ : There exists statistically significant difference in means of different groups of the variables at $1 \%$ level.

Data were expressed as mean \pm standard deviation. CKD: Chronic kidney disease, SBP: Systolic Blood Pressure, DBP: Diastolic Blood pressure, $\mathrm{Cr}$ : Creatinine, GFR: Glomerular filtration rate.

Table-1: Demographic and Biochemical data of study groups 


\begin{tabular}{|l|c|c|c|c|c|c|c|c|c|}
\hline Pearson's r & SBP & DBP & urea & Cr & GFR & MPV & PDW & PI count & P-LCR \\
\hline SBP & - & 0.116 & -0.050 & -0.086 & 0.062 & 0.151 & -0.185 & -0.013 & -0.057 \\
\hline DBP & 0.116 & - & 0.117 & 0.156 & -0.033 & -0.046 & -0.170 & -0.002 & 0.032 \\
\hline urea & -0.050 & 0.117 & - & 0.218 & -0.143 & 0.045 & -0.134 & 0.096 & -0.059 \\
\hline Cr & -0.086 & 0.156 & 0.218 & - & -0.834 & -0.001 & 0.055 & -0.182 & 0.034 \\
\hline GFR & 0.062 & -0.033 & -0.143 & -0.834 & - & -0.089 & -0.032 & 0.167 & -0.033 \\
\hline MPV & 0.151 & -0.046 & 0.045 & -0.001 & -0.089 & - & -0.191 & 0.146 & 0.245 \\
\hline PDW & -0.185 & -0.170 & -0.134 & 0.055 & -0.032 & -0.191 & - & 0.101 & 0.078 \\
\hline PI count & -0.013 & -0.002 & 0.096 & -0.182 & 0.167 & 0.146 & 0.101 & - & 0.102 \\
\hline P-LCR & -0.057 & 0.032 & -0.059 & 0.034 & -0.033 & 0.245 & 0.078 & 0.102 & - \\
\hline
\end{tabular}

Note: Blue colour indicates positive correlation and red colour indicates negative correlation. The darkness of the shade represents the strength of correlation. In Group 2 the above table shows Positive Correlation between 1.P-LCR and MPV 2. SBP and MPV and Negative Correlation between 1.PDW and MPV 2.PDW and SBP 3.Platelet Count and Creatinine.

Table-2: Correlation of the variables-Group 2

\begin{tabular}{|l|c|c|c|c|c|c|c|c|c|}
\hline Pearson's r & SBP & DBP & urea & Cr & GFR & MPV & PDW & PI count & P-LCR \\
\hline SBP & - & 0.374 & 0.334 & 0.135 & -0.081 & 0.336 & -0.122 & -0.045 & 0.327 \\
\hline DBP & 0.374 & - & 0.447 & -0.031 & 0.011 & 0.269 & -0.339 & -0.473 & -0.071 \\
\hline urea & 0.334 & 0.447 & - & 0.290 & -0.299 & 0.081 & 0.020 & -0.006 & -0.099 \\
\hline Cr & 0.135 & -0.031 & 0.290 & - & -0.946 & -0.066 & -0.012 & 0.069 & 0.082 \\
\hline GFR & -0.081 & 0.011 & -0.299 & -0.946 & - & 0.057 & 0.003 & -0.052 & -0.095 \\
\hline MPV & 0.336 & 0.269 & 0.081 & -0.066 & 0.057 & - & -0.181 & -0.071 & -0.189 \\
\hline PDW & -0.122 & -0.339 & 0.020 & -0.012 & 0.003 & -0.181 & - & 0.171 & 0.061 \\
\hline PI count & -0.045 & -0.473 & -0.006 & 0.069 & -0.052 & -0.071 & 0.171 & - & 0.374 \\
\hline P-LCR & 0.327 & -0.071 & -0.099 & 0.082 & -0.095 & -0.189 & 0.061 & 0.374 & - \\
\hline
\end{tabular}

Note: Blue color indicates positive correlation and red color indicates negative correlation. The darkness of the shade represents the strength of correlation. In Group 1 the above table shows Positive Correlation between 1. Urea and DBP 2.SBP and MPV 3.Urea and SBP 4.P-LCR and SBP 5.Platelet count and P-LCR 6.MPV and DBP and Negative Correlation between 1. Platelet Count and DBP 2.PDW and DBP.

Table-3: Correlation of the variables-Group 3

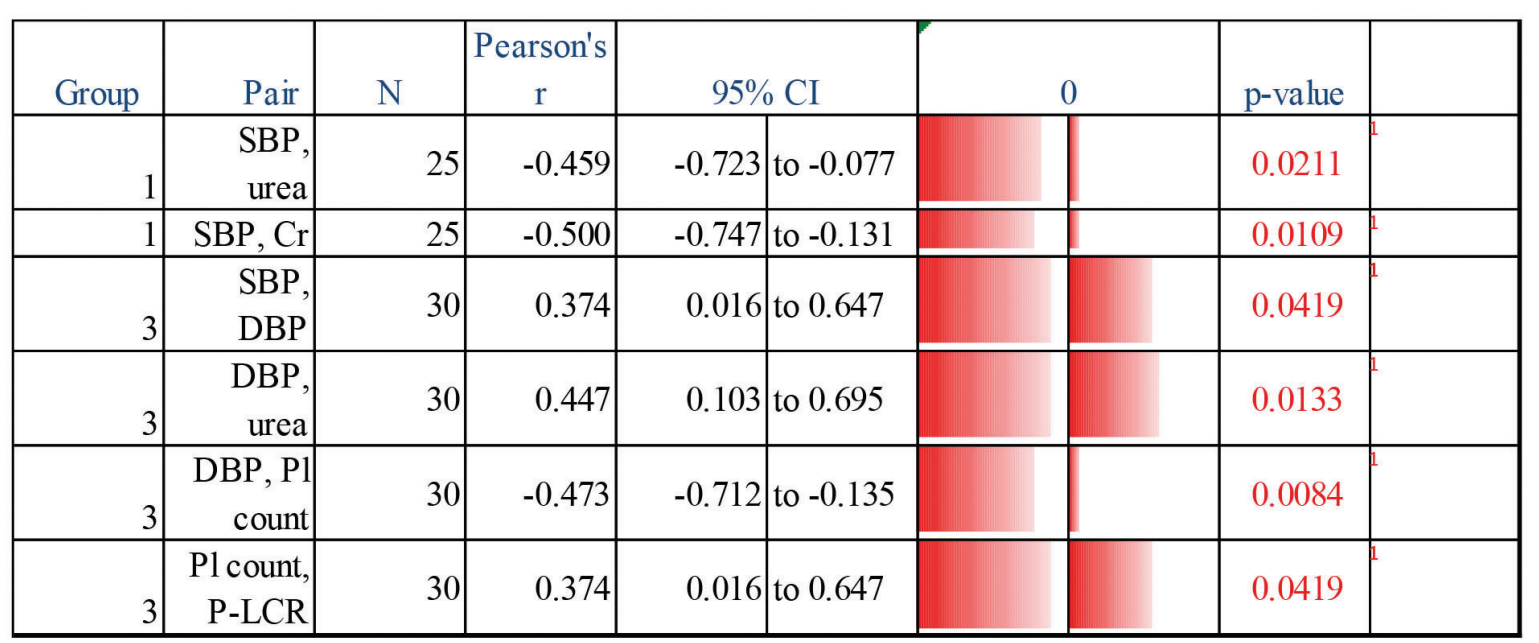

1Reject the null hypothesis in favour of the alternative hypothesis at the $5 \%$ significance level, i.e. the two variables are correlated $5 \%$ level of significance.

Table 4:Significance of correlation of paired variables

\section{DISCUSSION}

Platelet parameters were found to be significantly altered in chronic inflammatory conditions like chronic kidney disease and hence more pronounced in the state of haemodialysis. Because platelets have been identified as integrators of inflammatory mediators and endothelial cells. In this study, the platelet count was not showing any difference in group 1 in comparison to group 2. It was lower in group 3 than other two groups but which was not statistically significant even though increased platelet e-granulation during haemodialysis. This was in discordance with Schoorl et al ${ }^{11}$ who observed that CKD patients of stage 5 on maintenance 
HD had lower range of platelet count. Similarly In study by Lokesh S et al ${ }^{12}$ the average platelet count was found to be Decreased among patients on haemodialysis as compared to Controls which was found to be statistically significant . MPV reflects the average platelet size, most extensively studied platelet parameter as a pro thrombotic in chronic inflammatory conditions also an indicator of platelet activation and function, which can be useful to assess the prognosis of cardiovascular and cerebrovascular disease in CKD. Larger platelets are highly active and release more chemical mediators that are thrombotic factors. ${ }^{13}$ Increased $\mathrm{MPV}$ is an independent risk factor for cardiovascular disease in CKD. ${ }^{14,15}$ In this study, there was significant difference in MPV between the groups. When compared with control group with other two groups there was a statistically highly significant increase in MPV were noted. Koroglu et al. observed similarly a high MPV in CKD stage3 to 5 patients and inferred that MPV can be used as a biomarker to estimate cardiovascular risk in patients on haemodialysis. But at the same time MPV is minimally decreased in stage 5 kidney disease when compare to stages 3 and 4,may be due to the clearance of uremia better in case of haemodialysis and that difference is not statistically significant. Ju et al reported that there is a negative linear correlation between stages of $\mathrm{CKD}$ and MPV. ${ }^{14}$ Bilen et al, in their study, conducted with 50 patients with CKD stage 5 and 50 patients of Stage 3-4 $\mathrm{CKD}$, reported that there are no differences between the groups for MPV. ${ }^{16}$

The PDW is calculated mathematically from the platelet frequency distribution and is a measure of the variability of platelet sizes. As a result, when there is a variation in size from small to large, that will influence the PDW. Combined with the MPV, it can point out the possibility of enlarged platelets or even fragments of megakaryocyte, which are conditions with clinical significance in the risk of cardiovascular disease. PDW was significantly higher in group 2 and group 3 than with group 1 in our study. In addition, the difference between group 2 and 3 was not significant. Under healthy conditions, there is a direct relationship between MPV and PDW; both usually change in the same direction. ${ }^{17}$ These results are in match with MVP, having the common denominator of platelet activation could explain the same direction of change in our study.

L-PCR, Indicator of larger (> $12 \mathrm{fL}$ ) circulating platelets, was significantly lower in group 3 in comparison with group 1 and group 2, but in comparison to group 1 and group 2 the difference was not significant. Koroglu et al observed that P-LCR falls significantly in thrombocytosis but in our study, these happens in the setting of no significant change in platelet count. Schoorl M et al, in his study observed a similar decreased mean L-PCR in patient group of haemodialysis. ${ }^{18}$

\section{CONCLUSION}

In our study the MPV as an indicator of increased platelet activity was significantly increased in CKD stage 3,4 and 5 , when compared to general population, but at the same time it was NOT significantly altered by Haemodialysis. Similarly, PDW also significantly increased in CKD stage 3, 4 and 5.P-LCR was significantly lower in CKD stage 5 on maintenance Haemodialysis, might be due to increased production of platelets even in the setting of no significant thrombocytopenia.

\section{REFERENCES}

1. K.Okrainee, D.K. Banerjee,M.J. Eisenberg, Coronary artery disease in the developing world, Am. Heart J. 2004;148(5):7-15.

2. P.J. Best, D.N. Reddan, P.B. Berger, L.A. Szczech, P.A. McCullough, R.M. Califf,Cardiovascular disease and chronic kidney disease: insights and an update, Am.Heart J. 2004;148(6): 230-242.

3. M.P. Schlaich, F. Socratous, S. Hennebry, N. Eikelis, E.A. Lambert, N.Straznicky, M.D.Esler, G.W.Lambert, Sympathetic activation in chronic renal failure, J. Am. Soc.Nephrol. 2009;20(1):933-939.

4. C. Zoccali, Cardiorenal risk as a new frontier of nephrology: research needs andareas for intervention, Nephrol. Dial. Transplant. 2002;17(5):50-54.

5. Leader A, Pereg D, Lishner M. Are platelet volume indices ofclinical use? A multidisciplinary review. Ann Med 2012;44(5):805-816.

6. Bath PM, Butterworth RJ. Platelet size: Measurement, physiologyand vascular disease. Blood Coagul Fibrinolysis 1996;7(4):157-161.

7. Pal R, Bagarhatta R, Gulati S, Rathore M, SharmaN. Mean platelet volume in patients with acutecoronary syndromes: a supportive diagnosticpredictor. J Clinical Diagnostic Res. 2014;8(8):1-4.

8. Murat S, Duran M, Kalay N, GunebakmazO,Akpek M, Doger $\mathrm{C}$ et al. Relation between meanplatelet volume and severity of atherosclerosis inpatients with acute coronary syndromes. Angiology. 2012;64(2):131-6.

9. H.Uçar, M. Gür, N.Y. Koyunsever, T. Seker, C.Türkoğlu, O. Kaypakli, D.Y. Sahin, Z. Elbasan,M.Cayli, Mean platelet volume is independently associated with renal dysfunction in stable coronary artery disease, Platelets 2014;25 (4):274-278.

10. M. Schoorl, M. Schoorl, P.C. Bartels, Changes in platelet volume, morphology and RNA content in subjects treated with haemodialysis, Scand. J. Clin. Lab. Invest. 2008; 68:335-342.

11. Schoorl M, Nube M, Bartels P. Platelet depletion, platelet activation and coagulation during treatment with hemodialysis. Scandinavian J Clinical Lab Investigation, 2011; 71(3): 240-7.

12. Lokesh $\mathrm{S}$ et al. A comparative study of platelet parameters in end stage renal disease patients undergoing haemodialysis and healthy individualsInt J Adv Med. 2016;3(3):559-563.

13. Pereg D, Berlin T, Mosseri M. Mean platelet volume on admission correlates with impaired response to thrombolysis in patients with ST-elevation myocardial infarction. Platelets 2010; 21(3):117-21.

14. Güldiken B, Özkan H, Kabayel L. Mean platelet volume and peripheral blood count response in acute 15 chemic stroke. Trakya UnivTipFakDerg 2008;2(1):130-5.

15. Slavka G, Perkmann T, Haslacher $H$, et al. Mean platelet volume may represent a predictive parameter for overall vascular mortality and ischemic heart disease. ArteriosclerThrombVascBiol 2011;31(4): 1215-8 
16. Bilen Y, Cankaya E, Keles M, et al. Does decreased mean platelet volume predict inflammation in chronic renal failure, dialysis, and transplanted patients? Ren Fail 2014;36(1):69-72.

17. Vagdatli E, Gounari E, Lazaridou E, Katsibourlia E, Tsikopoulou F, Labrianou I. Platelet distribution width: a simple, practical and specific marker of activation of coagulation. Hippokratia 2010;14(3):28-32.

18. Schoorl M, Bartels $\mathrm{P}$. Changes in platelet volume, morphology and RNA content in subjects treated with haemodialysis. Scandinavian Journal of Clinical and Laboratory Investigation. 2008;68(4):335-42.

Source of Support: Nil; Conflict of Interest: None

Submitted: 16-05-2019; Accepted: 12-06-2019; Published online: 15-07-2019 